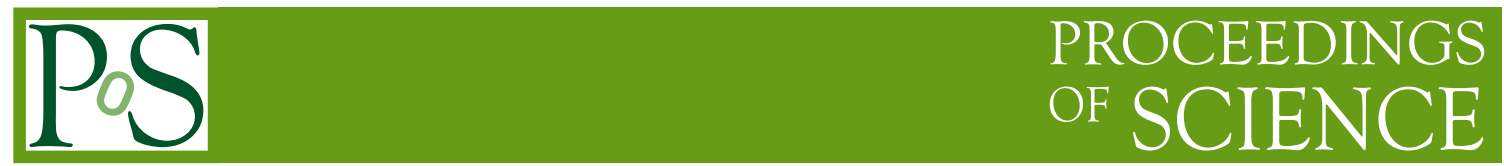

\title{
CP violation in beauty with the LHCb experiment
}

\author{
Alessandro Bertolin ${ }^{a, *}$ \\ ${ }^{a}$ Sezione INFN di Padova, \\ Via F. Marzolo 8, Padova, Italy \\ E-mail: alessandro.bertolin@pd.infn.it
}

These proceedings will review two recent LHCb measurements of the mass difference $\Delta m_{s}$ in the $B_{s}^{0}-\bar{B}_{s}^{0}$ system and present a LHCb combined $\Delta m_{s}$ value. Then recent measurements of the CKM angle $\gamma$ will be presented, followed by the correspondingly updated $\gamma$ combination result.

The Ninth Annual Conference on Large Hadron Collider Physics - LHCP2021

7-12 June 2021

Online

${ }^{*}$ Speaker 


\section{Measurements of the mass difference $\Delta m_{s}$ in the $B_{s}^{0}-\bar{B}_{s}^{0}$ system}

The most recent $\mathrm{LHCb}$ measurement of the mass difference between heavy and light mass eigenstates in the $B_{s}^{0}-\bar{B}_{s}^{0}$ system [1], $\Delta m_{s}$, takes advantage of the flavour specific nature of the decay $B_{s}^{0} \rightarrow D_{s}^{-} \pi^{+}$i.e. there are only oscillations between the two states decaying as $B_{s}^{0} \rightarrow D_{s}^{-} \pi^{+}$ and $\bar{B}_{s}^{0} \rightarrow D_{s}^{+} \pi^{-}$. The analysis exploits $6 \mathrm{fb}^{-1}$ of data collected during the LHC Run 2. A similar analysis using the LHC Run 1 data set has already been published [2]. The signal yield in Run 2 data is as large as 378,700 events and corresponds to a factor of 5.6 increase with respect to the Run 1 sample. The $\Delta m_{s}$ parameter is obtained from a fit to the background subtracted decay time distribution of the beauty meson candidates. This distribution is shown by the upper left plot in Fig. 1. Three components are shown separately: the untagged candidates and the candidates tagged as $B_{s}^{0}$ or $\bar{B}_{s}^{0}$. The continuous lines correspond to the values obtained from the central values of the fitted parameters. Given the huge signal sample recorded by $\mathrm{LHCb}$, the oscillation pattern for the tagged candidates is spectacular. This analysis establishes $\Delta m_{s}=17.7683 \pm 0.0051$ (stat.) \pm 0.0032 (syst.) $\mathrm{ps}^{-1}$.

Also the decay mode $B_{s}^{0} \rightarrow D_{s}^{-} \pi^{+} \pi^{+} \pi^{-}$can be similary used for a $\Delta m_{s}$ measurement. The LHCb measurement in this case [3] uses the full statistics available in Run 1 and Run 2, $9 \mathrm{fb}^{-1}$. The signal yield, 148,000 events, is smaller but comparable to the $B_{s}^{0} \rightarrow D_{s}^{-} \pi^{+}$analysis. The decay time distribution for candidates tagged as $B_{s}^{0}$ or $\bar{B}_{s}^{0}$ is shown by the upper right plot in Fig. 1. The continuous lines correspond to the values obtained from the central values of the fitted parameters. This analysis establishes $\Delta m_{s}=17.757 \pm 0.007$ (stat.) \pm 0.008 (syst.) $\mathrm{ps}^{-1}$.

A LHCb $\Delta m_{s}$ combination can be performed using 5 statistically independent measurement using the $D_{s}^{-} \pi^{+}[1,2], D_{s}^{-} \pi^{+} \pi^{+} \pi^{-}[3]$ and the $J / \psi K^{+} K^{-}[4,5]$ final states. The corresponding $\Delta m_{s}$ values are shown by the bottom plot in Fig. 1, as well as the combined value, represented by a vertical band. The all hadronic final state measurements have the best precision.

The average is found to be $\Delta m_{s}=17.7656 \pm 0.0057 \mathrm{ps}^{-1}$ to be compared with a lattice QCD plus sum rules theoretical prediction [6] of $18.4_{-1.2}^{+0.7} \mathrm{ps}^{-1}$.

Given the large theoretical uncertainty a much more accurate measurement of $\Delta m_{s}$ is very welcome.

\section{Measurements of the CKM angle $\gamma$}

The angle $\gamma$ is defined in terms of the elements of the CKM matrix as $\arg \left(-\frac{V_{u d} V_{u b}^{*}}{V_{c d} V_{c b}^{*}}\right)$. It is the only angle of the CKM unitarity triangle relevant for beauty mesons decays easily accessible at tree-level. Moreover the theoretical uncertainties affecting $\gamma$ measurements are negligible. Measurements of $\gamma$ with an accuracy of about $5^{\circ}$ can be obtained considering the decay modes $B^{ \pm} \rightarrow D^{0}\left(\rightarrow K_{s}^{0} h^{+} h^{-}\right) K^{ \pm}$and $B^{ \pm} \rightarrow \bar{D}^{0}\left(\rightarrow K_{s}^{0} h^{+} h^{-}\right) K^{ \pm}$. The first is proportional to $V_{c b}$ and the second to $V_{u b}$, hence the sensitivity to $\gamma$. These decay modes have been used in a recent LHCb analysis [7] using an integrated luminosity of $9 \mathrm{fb}^{-1}$, the full Run $1+$ Run 2 statistics. The $\mathrm{CP}$ violation parameters are determined from measuring the $B^{-}$and $B^{+}$signal variation over the $m\left(K_{s}^{0}, h^{+}\right)$vs $m\left(K_{s}^{0}, h^{-}\right)$Dalitz plot, where the function $m(a, b)$ represents the invariant mass of the particles used as arguments. The only external input required is the averaged strong-phase difference between the $D^{0}$ and $\bar{D}^{0}$ decay amplitudes in regions of phase space, which is determined from CLEO and BESIII data. Results are presented in Fig. 3 by the black contours in the $\delta_{B}^{D K}$ 

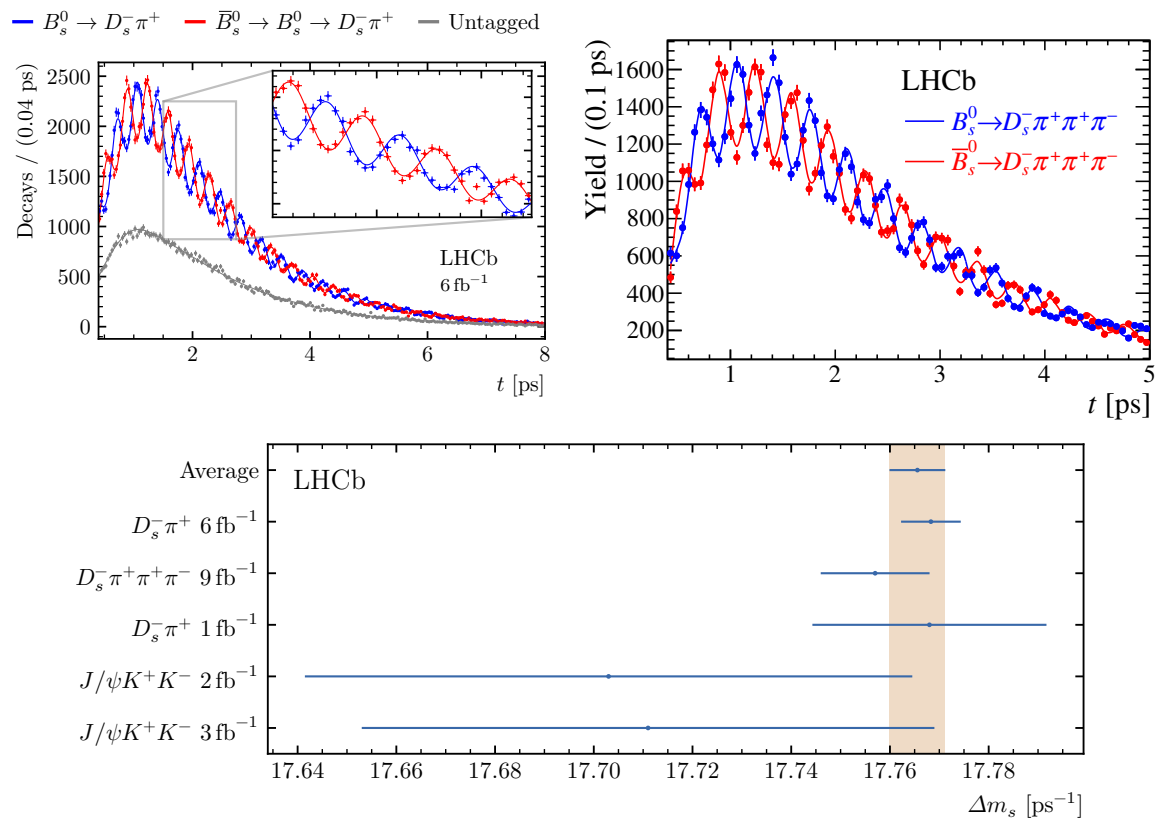

Figure 1: Decay time distributions observed in the analyses of $B_{s}^{0} \rightarrow D_{s}^{-} \pi^{+}$, upper left plot, and $B_{s}^{0} \rightarrow$ $D_{s}^{-} \pi^{+} \pi^{+} \pi^{-}$, upper right plot. The different components and continuous lines shown are explained in the text. A collection of statistically independent $\Delta m_{s}$ measurements is shown in the lower plot together with their average represented as a vertical band.
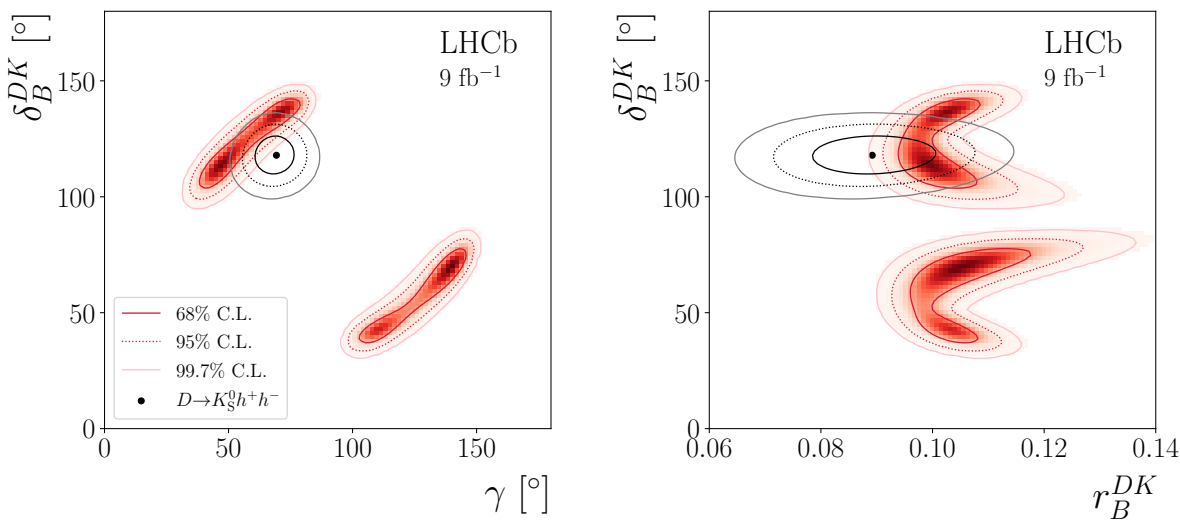

Figure 2: Allowed regions in the $\delta_{B}^{D K}$ vs $\gamma$, left plot, and the $\delta_{B}^{D K}$ vs $r_{B}^{D K}$, right plot, planes from the two time independent analyses described in the text.

vs $\gamma$ plane, left plot, and in the $\delta_{B}^{D K}$ vs $r_{B}^{D K}$ plane, right plot. Here $r_{B}^{D K}$ is the ratio between the suppressed and the favoured decay amplitudes and $\delta_{B}^{D K}$ the corresponding strong phase difference.

The decay modes $B^{ \pm} \rightarrow D^{0}\left(\rightarrow h^{+} h^{-}\right) K^{ \pm}$and $B^{ \pm} \rightarrow \bar{D}^{0}\left(\rightarrow h^{+} h^{-}\right) K^{ \pm}$also provide strong constraints on $\gamma$. The first is proportional to $V_{c b}$ and the second to $V_{u b}$. The LHCb analysis using these decay modes [8] is based on an integrated luminosity of $9 \mathrm{fb}^{-1}$, as was the previously presented one. The measured partial decay rates are related to the underlying physical parameters. To exploit 

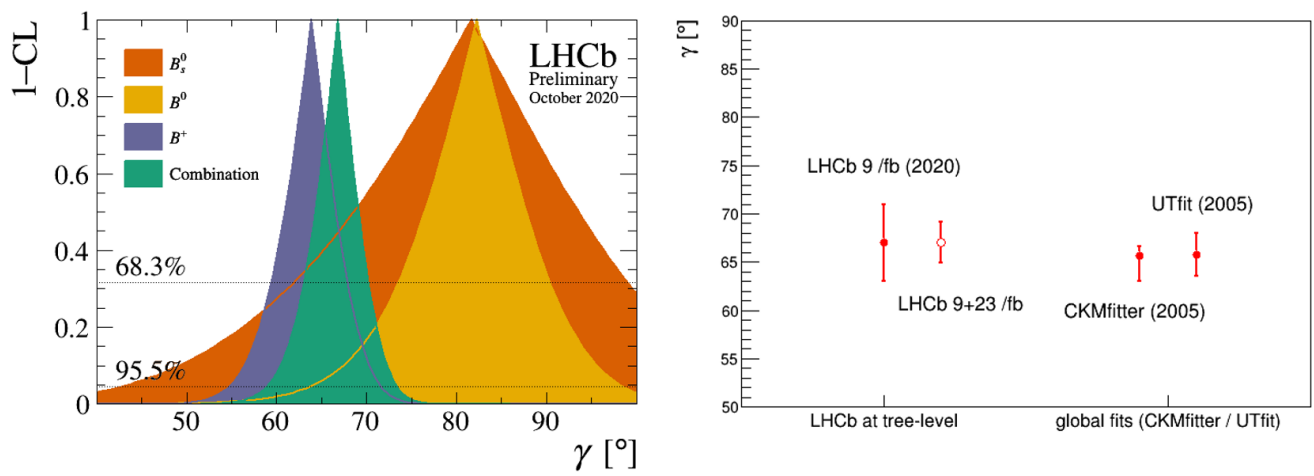

Figure 3: One dimensional 1-CL scan showing the result of the LHCb $\gamma$ combination, left plot. The components corresponding to the various $B$-meson species are also reported. Comparison of the $\mathrm{LHCb}$ $\gamma$ combination result, obtained using $9 \mathrm{fb}^{-1}$, with the CKMfitter and UTfit predictions, right plot. An extrapolation of the $\mathrm{LHCb}$ result to $9+23 \mathrm{fb}^{-1}$ is also shown.

as much as possible the available data set the neutral charm mesons are reconstructed in the $K^{ \pm} \pi^{\mp}$ $K^{+} K^{-}$and $\pi^{+} \pi^{-}$final states. Moreover the partially reconstructed decays $B^{ \pm} \rightarrow D^{*}\left(\rightarrow D \pi^{0} / \gamma\right) K^{ \pm}$ are included, where $D^{*}$ stands for $D^{0 *}$ or $\bar{D}^{0 *}$, similarly $D$ stands for $D^{0}$ or $\bar{D}^{0}$ and the neutral particle, $\pi^{0}$ or $\gamma$, is not reconstructed. These partially reconstructed decays are used for the first time with these decay modes. All CP observables of these decay modes are measured with world-leading precision. The results in the $\delta_{B}^{D K}$ vs $\gamma$ plane and in the $\delta_{B}^{D K}$ vs $r_{B}^{D K}$ plane are represented by the red contours in Fig. 2. A two-fold ambiguity is apparent. This ambiguity can be solved using the results presented in the previous paragraph, leading to a $\gamma$ value of $(61.8 \pm 4.0)^{\circ}$.

The best accuracy on $\gamma$ can be obtained by combining measurements performed on $B^{+}, B^{0}$ and $B_{s}^{0}$ mesons, using time dependent or time integrated analysis techniques and different final state topologies. At present, the LHCb $\gamma$ combination [9] uses input from $16 \mathrm{LHCb}$ measurements directly sensitive to $\gamma$, and some auxiliary input parameters. Whenever possible these auxiliary parameters are taken from dedicated measurements, often performed by the LHCb collaboration. As already mentioned, other auxiliary inputs are strong-phase related parameters determined from CLEO and BESIII data or through charm mixing analyses. The left plot in Fig. 3 shows the 1-CL distribution separately for each $b$-meson type as well as the combination. The combination, resulting in $\gamma=(67 \pm 4)^{\circ}$, is driven by the $B^{+}$mesons results. The right plot compares the $\mathrm{LHCb}$ $\gamma$ combination from tree-level processes, as of 2020, with the global CKM fits predictions from CKMfitter [10] and UTfit [11]. The right plot also shows the precision expected from LHCb by adding to the $9 \mathrm{fb}^{-1}$ collected so far all the expected luminosity in 2022-2024, $23 \mathrm{fb}^{-1}$. On a longer time scale $\mathrm{LHCb}$ will reach an accuracy below one degree [12]. This comparison between tree-level determinations and the value inferred from global CKM fits is of fundamental importance because it could indicate physics beyond the SM due, for example, to new particles or mediators being exchanged in loops. 


\section{References}

[1] LHCb collaboration, R. Aaij et al., Precise determination of the $B_{s}^{0}-\bar{B}_{s}^{0}$ oscillation frequency, submitted to Nature Physics, arXiv:2104.04421

[2] LHCb collaboration, R. Aaij et al., Precision measurement of the $B_{s}^{0}-\bar{B}_{s}^{0}$ oscillation frequency in the decay $B_{s}^{0} \rightarrow D_{s}^{-} \pi^{+}$, New J. Phys. 15 (2013) 053021

[3] LHCb collaboration, R. Aaij et al., Measurement of the CKM angle $\gamma$ and $B_{s}^{0}-\bar{B}_{s}^{0}$ mixing frequency with $B_{s}^{0} \rightarrow D_{s}^{ \pm} h^{ \pm} \pi^{ \pm} \pi^{\mp}$ decays, JHEP 03 (2021) 137

[4] LHCb collaboration, R. Aaij et al., Precision measurement of CP violation in $B_{s}^{0} \rightarrow J / \psi K^{+} K^{-}$ decays, Phys. Rev. Lett. 114 (2015) 041801

[5] LHCb collaboration, R. Aaij et al., Updated measurement of time-dependent CP-violating observables in $B_{s}^{0} \rightarrow J / \psi K^{+} K^{-}$decays, Eur. Phys. J. C79 (2019) 706, Erratum ibid. C80 (2020) 601

[6] L. Di Luzio, M. Kirk, A. Lenz, and T. Rauh, $\Delta M_{s}$ theory precision confronts flavour anomalies, JHEP 12 (2019) 009

[7] LHCb collaboration, R. Aaij et al., Measurement of the CKM angle $\gamma$ in $B^{ \pm} \rightarrow D K^{ \pm}$and $B^{ \pm} \rightarrow D \pi^{ \pm}$decays with $D \rightarrow K_{s}^{0} h^{+} h^{-}$, JHEP 02 (2021) 169

[8] LHCb collaboration, R. Aaij et al., Measurement of CP observables in $B^{ \pm} \rightarrow D^{(*)} K^{ \pm}$and $B^{ \pm} \rightarrow D^{(*)} \pi^{ \pm}$decays using two-body $D$ final states, JHEP 04 (2021) 081

[9] LHCb collaboration, R. Aaij et al., Updated LHCb combination of the CKM angle $\gamma, \mathrm{LHCb}-$ CONF-2020-003

[10] CKMfitter group, J. Charles et al., CP violation and the CKM matrix: Assessing the impact of the asymmetric B factories, Eur. Phys. J. C41 (2005) 1

[11] UTfit collaboration, M. Bona et al., The 2004 UTfit collaboration report on the status of the unitarity triangle in the standard model, JHEP 07 (2005) 028

[12] LHCb collaboration, I. Bediaga et al., Physics case for an LHCb Upgrade II - Opportunities in flavour physics, and beyond, in the HL-LHC era, arXiv:1808.08865 